УДК 681.515

DOI https://doi.org/10.32838/2663-5941/2019.3-1/32

Улицкая Е.O.

Одесский национальный политехнический университет

\title{
Лысюк А.П.
}

Одесский национальный политехнический университет

Костюкова О.Н.

Одесский национальный политехнический университет

\section{РАЗРАБОТКА И ИССЛЕДОВАНИЕ КОМПЬЮТЕРНО- ИНТЕГРИРОВАННОЙ СИСТЕМЫ УПРАВЛЕНИЯ УРОВНЕМ ВОДЫ С РАЗРАБОТКОЙ УЧЕБНОГО СТЕНДА}

Статья посвящена разработке АСР уровня воды в емкости с возможностью изменения законов регулирования, задания, а также нагрузки в режиме реального времени на базе платформы Аrdиіпо с визуализачией регулируемой величины и управляющего воздействия.

Ключевые слова: уровнемер, управляющее воздействие, возмущающее воздействие, закон регулирования.

Постановка проблемы. Развитие учебнолабораторной базы для подготовки специалистов по компьютерным технологиям и автоматизации, оперативная адаптация лабораторных и практических занятий для изучения наиболее актуальных вопросов автоматизации являются задачами совершенствования системы подготовки кадров. Поиск путей совершенствования методических подходов в образовательной деятельности, как в целом, так и в практической составляющей обучения, является одной из важнейших задач высшей школы на современном этапе [3, с. 7]. Модернизация лабораторной базы для подготовки специалистов по автоматизации предполагает создание новых учебных лабораторий для исследования компьютерно-интегрированных систем управления уровня, что позволит сформировать необходимые практические навыки и выработать требуемые компетенции для будущей профессиональной деятельности студентов.

Анализ последних исследований и публикаций. Появление первых микропроцессоров ознаменовало начало новой эры в развитии микропроцессорной техники. Наличие в одном корпусе большинства системных устройств сделало микроконтроллер подобным обычному компьютеру. Раньше они назывались однокристальными микроЭВМ. Чтобы собрать устройство и микроконтроллер, необходимо знать основы схемотехники, устройство и работу конкретного процессора, уметь программировать на ассемблере и изготавливать электронную технику. В настоящее время все изменилось. Сейчас существует такое устройство, как проект Arduino [5, с. 55]. В одной из последних статей Р.А. Аджиева («Микроконтроллеры. Arduino и IDE среда разработки») описано начало создания Arduino, историческая справка и все положительные стороны данной платформы.

Сегодня существует множество микроконтроллеров и платформ для осуществления управления физическими процессами применительно к микропроцессорным комплексам. Большинство этих устройств объединяют разрозненную информацию о программировании и заключают ее в простую в использовании сборку. Фирма Arduino, в свою очередь, тоже упрощает процесс работы с микроконтроллерами, однако обеспечивает ряд преимуществ перед другими устройствами из-за простой и понятной среды программирования, низкой цены и множеством плат расширения. В статье В.С. Лободинова, С.Р. Пана, И.В. Пугачева, В.Н. Трофименкова, Я.Н. Тузка «Применение Arduino в учебном процессе» показано, что для преподавателей, студентов и любителей платформа Arduino стала основным элементом для исследования и решения задач в областях мехатроники, робототехники и автоматизации [5, с. 51].

Постановка задачи. Цель статьи - разработать учебно-лабораторный стенд исследования компьютерно-интегрированной системы управления уровнем воды в емкости для возможности использования в учебном процессе на практических занятиях на базе платформы Arduino, разра- 
ботать программное обеспечение для визуализации переходных процессов регулирования.

Изложение основного материала исследования. Принципиальная схема разрабатываемого стенда изображена на рис. 1. Установка состоит из двух емкостей, наполненных жидкостью, в каждой из которых установлен насос (управляющий 5 и возмущающий 4$)$. Ток зависит от напряжения питания (2,5-6 В), мощности 0.4-1.5 Вт, скорость перекачивания жидкости составляет до 2 л/мин, или 120 л/час. Материал корпуса - пластик, загерметизирован. Максимальная высота водяного столба - 0,4-1,1 м.

В емкости 1 поддерживается уровень жидкости с помощью насоса 5, который приводит к перекачиванию воды из емкости 2 в емкость 1. Установка может работать в двух режимах - авто и ручной, которые выбираются с помощью ИК - пульта управления 10. ИК-приемник и инфракрасный пульт дистанционного управления - самый распространенный и простой способ управления электронной аппаратурой. С помощью ИК-пульта также меняем задание, коэффициенты регулятора и осуществляем выбор закона регулирования. Устройство работает на расстоянии $40 \mathrm{M}$.

Насос 5 управляется с помощью MOSFET транзистора в ШИМ-режиме. Модуль (драйвер) силового MOSFET-транзистора используется для подключения мощной нагрузки на выход контроллера, работающей на постоянном токе. Отличие этого модуля от модуля реле в его высоком быстродействии и долговечности. Отсутствие механических контактов в данном модуле управления нагрузкой, по сравнению с электромагнитным реле, дает на несколько порядков большую надёжность и долговечность. Модуль имеет

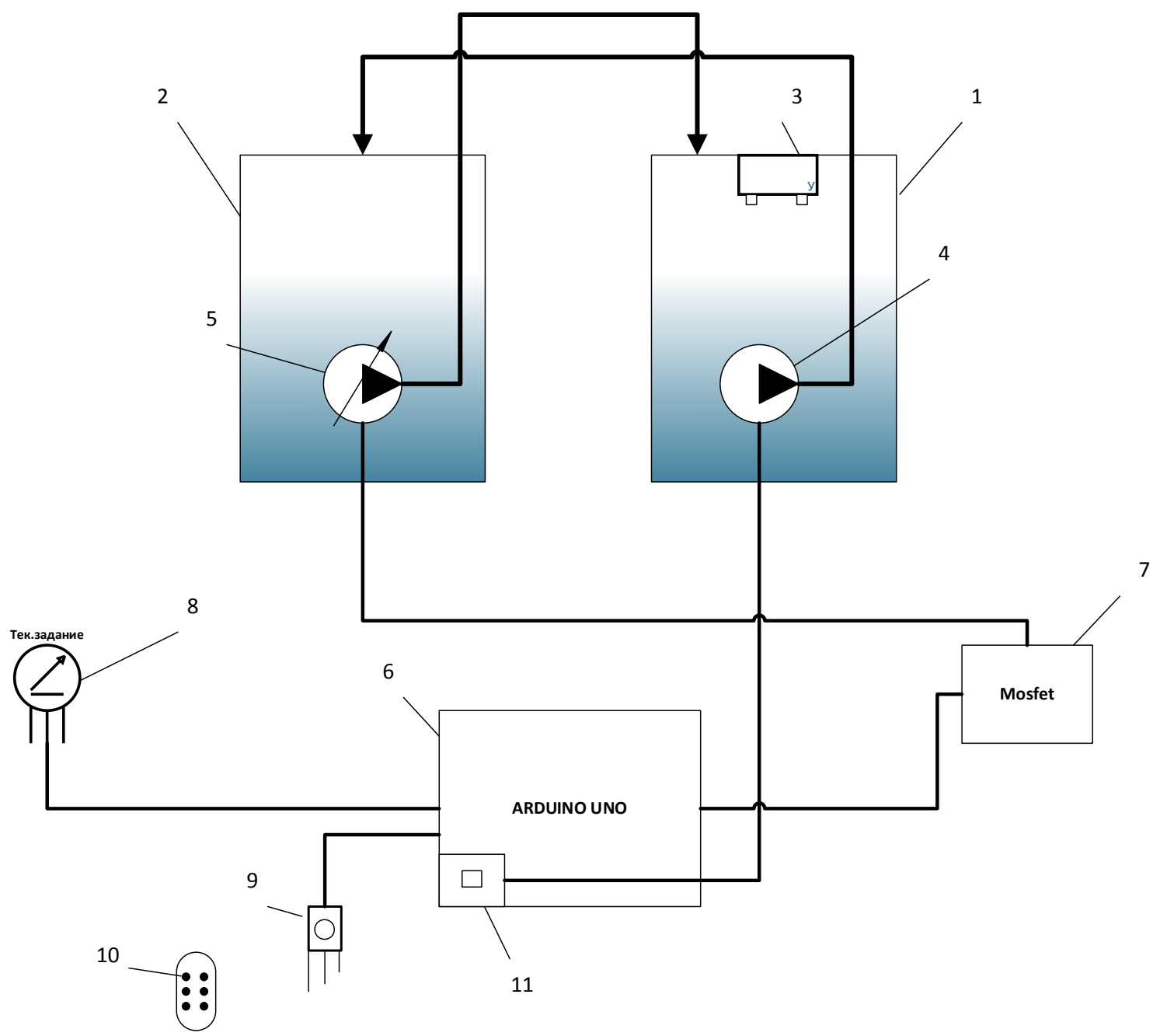

Рис. 1. Принципиальная схема установки:

1 - емкость с регулируемым уровнем; 2 - емкость слива лишней жидкости;

3 - ультразвуковой модуль НC-SR04; 4 - возмущающий насос, имитирующий потребление воды; 5 - управляющий насос; 6 - плата Ардуино UNO; 7 - транзистор; 8 - сервопривод;

9 - ИК-приемник; 10 - пульт управления; 11 - кнопка для изменения расхода воды в емкости 1. 
управляющее напряжение 3,3-5 B, напряжение на выходе 0-24 В и ток нагрузки до 5 А.

Измерение уровня в емкости 1 проводится с помощью ультразвукового модуля HC-SR04. Устройство посылает 8 импульсов звуковых волн с частотой 40 кГц и принимает отраженную волну. Далее измеряется временная задержка между отправленным и принятым сигналом и происходит вычисление расстояния по формуле $\mathrm{D}=\mathrm{TS} / 2$, где $\mathrm{D}$ - это расстояние, $\mathrm{T}$ - временная задержка и $\mathrm{S}$ - скорость звукового сигнала.

Датчик имеет разрешение $0,3 \mathrm{~cm}$, а диапазон измерения от 2 до $500 \mathrm{~cm}$.

Поддержание уровня в резервуаре 1 может осуществляться одним из трех законов регулирования - П, ПИ и релейным. Весь процесс реализован на платформе Arduino Uno. Arduino Uno - этот контроллер построен на платформе ATmega328, имеющей 14 цифровых вход/выходов (6 из которых могут использоваться как выходы ШИМ), 6 аналоговых входов, кварцевый генератор 16 МГц, разъем USB, силовой разъем, разъем ICSP и кнопку перезагрузки [6, с. 152].

С помощью разработанного стенда получены экспериментальные кривые разгона (рис. 2) по каналу управления и по каналу возмущения.

Передаточные функции объекта по каналу управления и возмущения являются интегрирующими звеньями. А коэффициенты передаточных функций, определенные по кривым разгона имеют вид:

$$
\mathrm{W}_{\text {упр }}=\frac{\mathrm{k}}{p}=\frac{0,004}{p}
$$

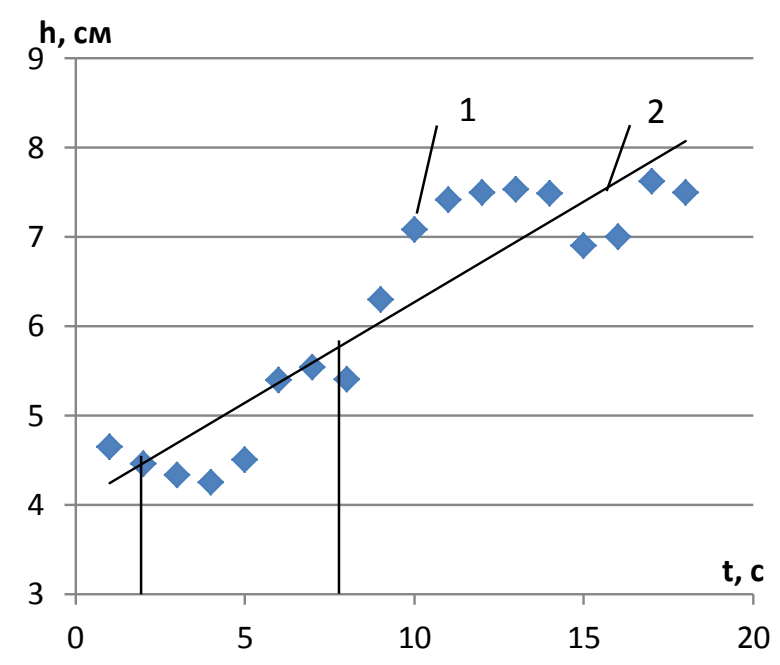

a

Рис. 2. Кривая разгона объекта при изменении управляющего воздействия (а) и возмущающего воздействия (б): 1 - экспериментальные точки; 2 - аппроксимирование точки (точки сглаживания)

$$
\mathrm{W}_{\text {возм }}=\frac{\mathrm{k}}{p}=\frac{-0,004}{p}
$$

После получения передаточных фунцкий объекта были синтезированы цифровые П и ПИ законы регулирования, а также реализован релейный регулятор [9, с. 40].

Разработанный стенд (рис. 3) функционирует согласно следующему алгоритму:

При нажатии кнопки 11 изменяется расход воды из емкости 1, в которой поддерживается уровень жидкости, происходит включение насоса 4, который приводит к перекачиванию воды из емкости 1 в емкость 2. Результат представлен на рис. 5, где показано изменение уровня, в соответствии с включенным регулятором - П, ПИ и релейный, в емкости 1 при удержании кнопки 11 в течении 10 с. На рис. 4 мы можем наблюдать, что при действии возмущения регулятор выводит значение уровня на заданное.

Разработанный стенд позволяет получать графики переходных процессов регулирования, демонстрирует отличие действия П, ПИ и релейного закона управления [7, с. 179], в составе АCP уровня (рис. 4,5 ).

При увеличении задания с помощью ИК-пульта 10 регулятор выводит уровень на заданный (14 см), что показано на рис. 4.

При работе с платой Arduino результат вычисления выводится на Serial Monitor. Но это не единственная возможность для получения данных на экране. Приложение Processing имеет очень много возможностей, в том числе и библиотеку Serial,

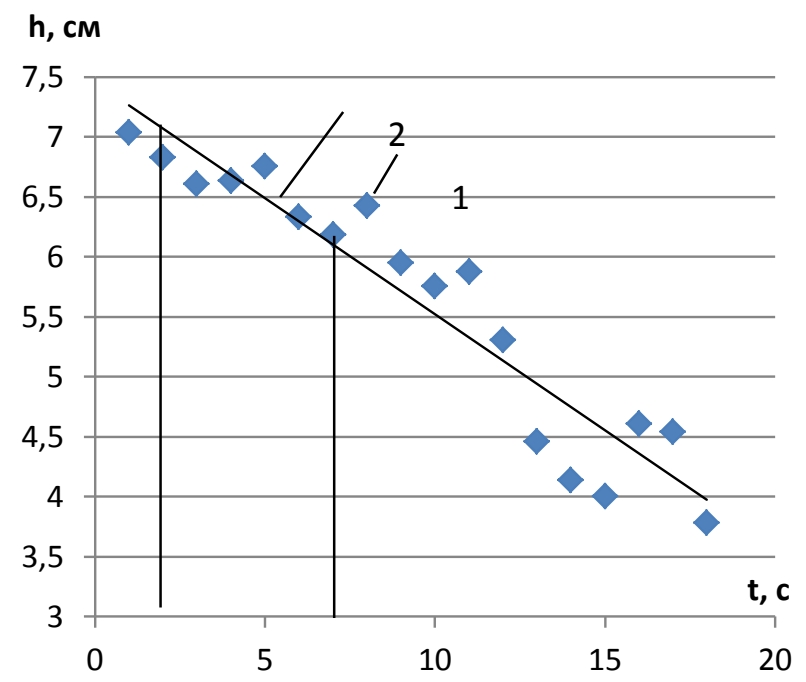

б 


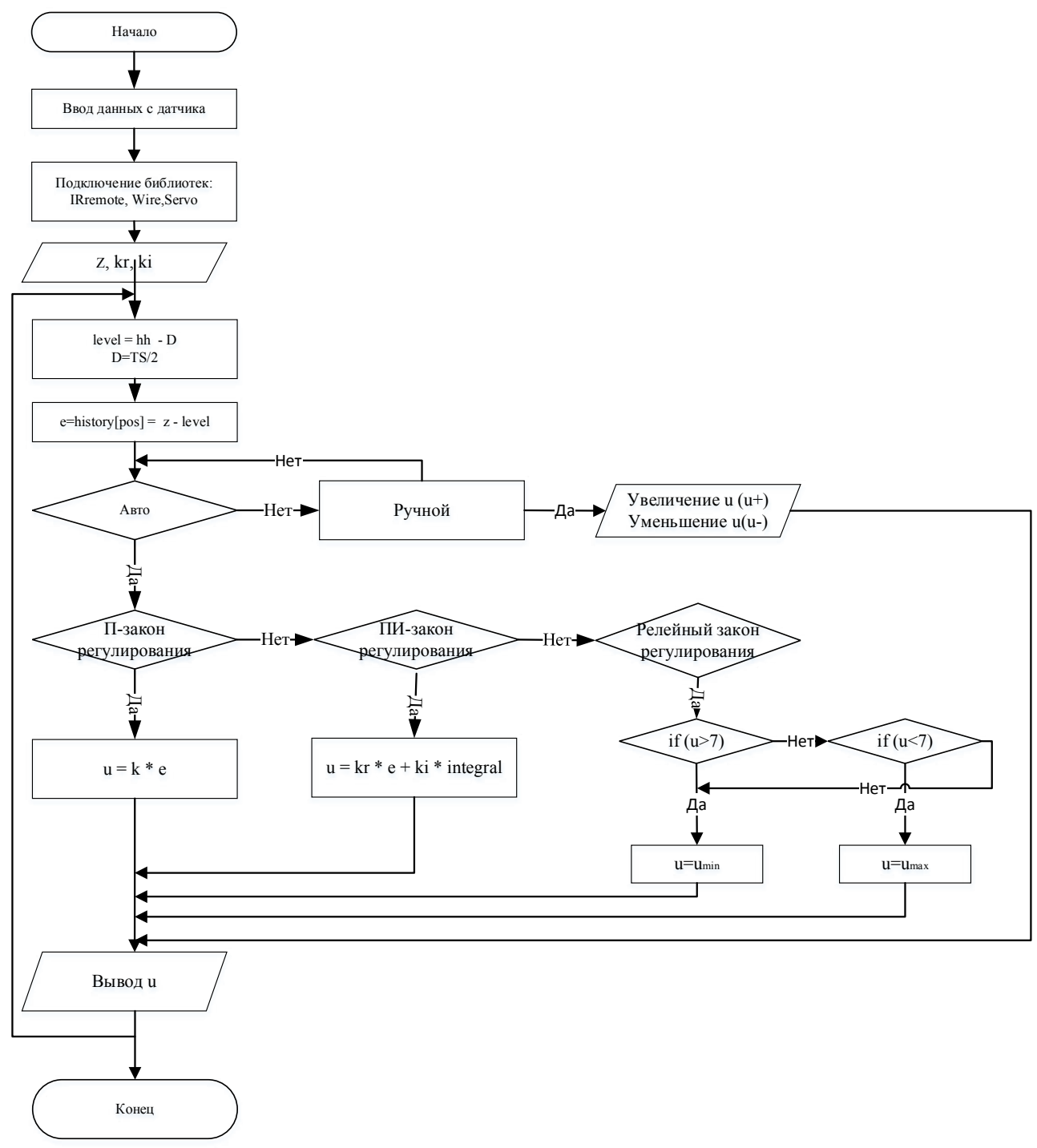

Рис. 3. Структурна схема установки

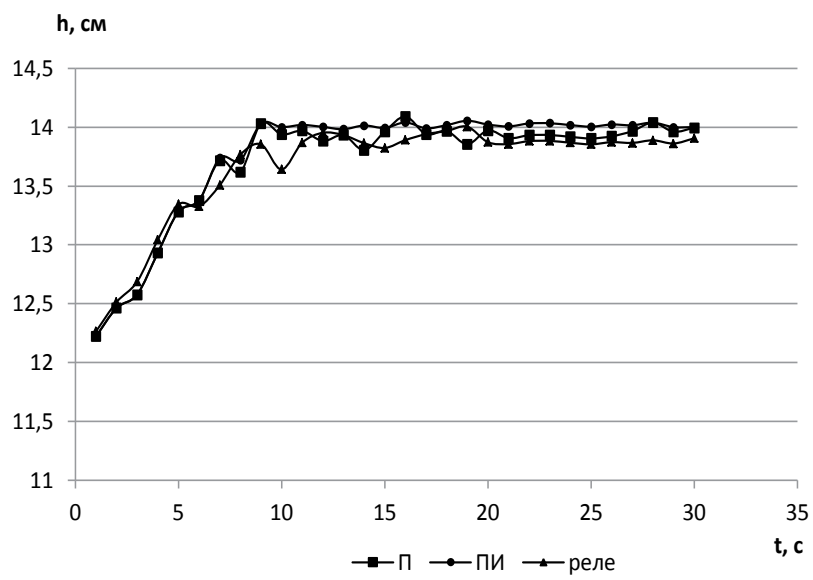

Рис. 4. Переходной процесс регулирования уровня при изминении задания с использованием разных видов регулятора

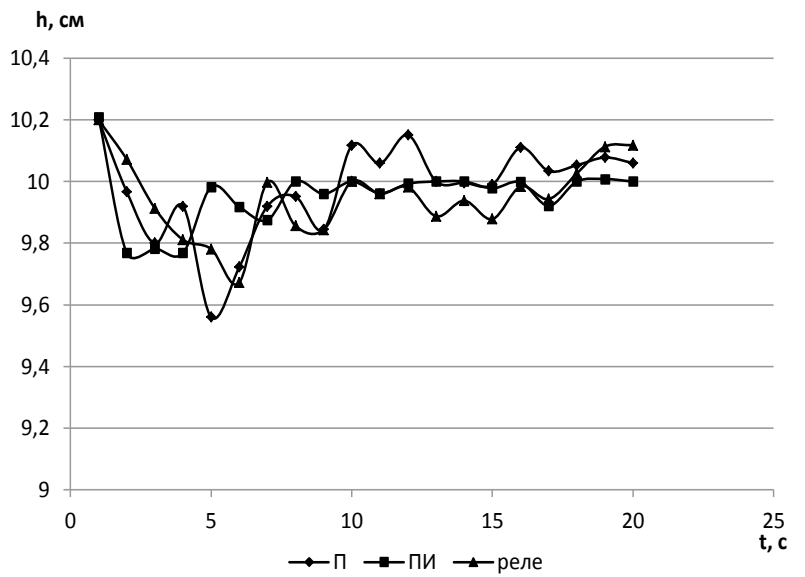

Рис. 5. Переходной процесс регулирования уровня с разными регуляторами при действии возмущения 
поэтому возможна передача данных между платой и Processing. Processing - это язык программирования для создания интерактивных визуальных эффектов, базируется на графических возможностях языка Java и использует его упрощённый синтаксис, лёгкий и быстрый инструментарий.

Для разработанного учебного стенда была написана программа в Processing, с помощью которой мы можем наблюдать изменение уровня и возмущения в режиме реального времени, также выводим значения заданного и текущего уровня.

На рис. 6 мы можем наблюдать процесс регулирования уровня.

C помощью приложения Processing peализовано графическое представление данных с датчика уровня, а также управляющего воздействия и задания регулятора.

Выводы. Таким образом, платформу Arduino можно использовать в учебном процессе для создания лабораторных установок. Была разработана АCP уровня воды в емкости с возможностью изменения законов регулирования, задания, а также нагрузки в режиме реального времени, а также разработано программное обеспечение

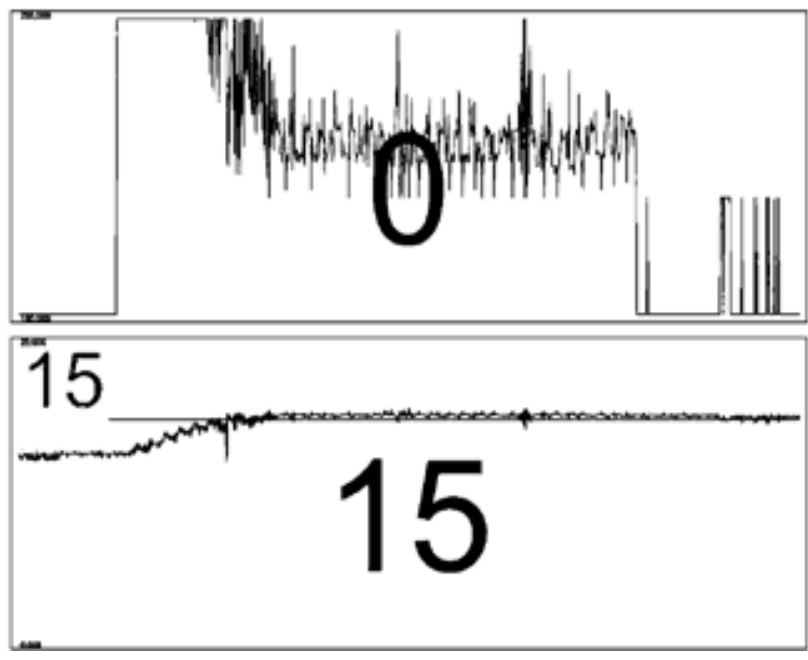

Рис. 6. Вывод в Processing

для визуализации переходных процессов регулирования.

Обучение студентов с использованием данной лабораторной установки позволит сформировать необходимые практические навыки и выработать требуемые компетенции для будущей профессиональной деятельности студентов.

\section{Список литературы:}

1. Улицкая Е.О., Бундюк А.Н. Разработка алгоритма для расчёта статики когенерационной энергетической установки. Холодильная техника и технология. 2013. № 3. С. 34-40.

2. Лисюк А.П., Белова Н.A. Genetic algorithms application to decide the issue of single-dimensional cutting optimization. Automation of technological and business processes. 2016. № 2. Т. 8. Р. 4-8.

3. Данилов А.Н., Кон Е.Л., Кон Е.М., Южаков А.А. Модель многоканального управления учебным процессом высшей школы. Открытое образование. 2012. № 2. С. 7-11.

4. Онлайн курс по Ардуино на базе простого стартового набора. Geektimes. URL: https:/geektimes.ru/ post/279860/ (дата обращения: 28.04.2019)

5. Лободинов В.С., Пан С.Р., Пугачев И.В., Трофименко В.Н., Тузко Я.Н. Применение Arduino в учебном процессе. Молодой исследователь Дона. 2019. № 1(16). С. 51-55.

6. Байда А.С. Использование платформы Arduino при подготовке специалистов автомобильной отрасли. Научно-методический электронный журнал «Концепт». 2016. № 5 (май). С. 150-156.

7. Ложечников В.Ф., Михайленко В.С. Методи побудови адаптивних систем управління. Автоматика. Автоматизация. Электротехнические комплексы и системы. 2009. № 2. С. 174-179.

\section{РОЗРОБКА І ДОСЛІДЖЕННЯ КОМП'ЮТЕРНО-ІНТЕГРОВАНОЇ СИСТЕМИ УПРАВЛІННЯ РІВНЕМ ВОДИ З РОЗРОБКОЮ НАВЧАЛЬНОГО СТЕНДУ}

Стаття присвячена розробці АСР рівня води в ємності з можливістю зміни законів регулювання, завдання, а також навантаження в режимі реального часу на базі платформи Агdиіпо з візуалізацією регульованої величини і дї, щуо управляє.

Ключові слова: рівнемір, управляюча дія, збурюючий вплив, закон регулювання.

\section{THE COMPUTER INTEGRATED CONTROL SYSTEM OF WATER LEVEL WITH DEVELOPMENT OF THE EDUCATIONAL STAND}

Article is devoted to development of ASR of water level in capacity with a possibility of change of laws of regulation, a task and also loading in real time on the basis of the Arduino platform with visualization of adjustable size and the operating influence.

Key words: level gauge, operating influence, revolting influence, law of regulation. 